\title{
Optimal Unit Commitment Problem Considering Stochastic Wind Energy Penetration
}

\author{
Khalid Alqunun \\ College of Engineering \\ University of Ha'il, Saudi Arabia \\ khalid.alqunun@gmail.com
}

\begin{abstract}
Wind energy has attracted much attention as a clean energy resource with low running cost over the last decade, However, due to the unpredictable nature of wind speed, the Unit Commitment (UC) problem including wind power becomes more difficult. Therefore, engineers and researchers are required to seek reliable models and techniques to plan the operation of thermal units in presence of wind farms. This paper presents a new attempt to solve the stochastic UC including wind energy sources. In order to achieve this, the problem is modeled as a chance-constrained optimization problem. Then, a method based on the here-and-now strategy is used to convert the uncertain power balance constraint into a deterministic constraint. The obtained deterministic problem is modeled using Mixed Integer Programing (MIP) on GAMS interface whereas the CEPLEX MIP solver is employed for its solution.
\end{abstract}

Keywords-stochastic optimization; mixed-integer programming; unit commitment; wind energy sources

\section{INTRODUCTION}

\section{A. Research Background}

Wind power generation has attracted much attention due to the emergence of energy crises and the related environmental issues. Thus, engineers and researchers in power systems have encountered new challenges due to the intermittent and stochastic nature of Wind Power (WP). One of the main challenges is to study the Unit Commitment (UC) when Wind Energy Sources (WESs) are incorporated into the main power network. The UC problem aims to determine which units have to be committed/uncommitted from the economic perspective [1]. The production levels of the committed units must be specified in order to meet the predicted load at a minimum total production cost over a planning horizon varying from one day to one week. In general, most of the operation costs include the start-up and shut-down costs [2]. The minimization of the operation cost is affected by several operating constraints, which can limit the search space. Power balance constraint, generation limits, spinning reserve constraints, minimum uptime/down-time constraints, unit initial status, and ramp rate limits of units are the most used constraints in the UC problem formulation [3-4]. The decision variables for the UC problem consist of both states of units, which are based on binary/integer variables (i.e. 1 when the unit is $\mathrm{ON}$ and 0 when the unit is OFF) and the output power of the committed units, which are continuous/real variables. For a long time, the UC problem has been mainly formulated as a mixed-integer optimization problem [5-9]. Recently, the issues of climate change have begun to be a global concern and some countries have made immediate decisions to decrease their gas emissions [10]. On the other hand, WESs are characterized by the lowest generation cost and the largest available energy amongst all renewable energy sources [11]. Due to the aforementioned reasons, some countries are committed to using WES. The integration of WES into power grids is usually accompanied by several difficulties, such as the stochastic availability of wind energy [12]. Therefore, scientific researches and technological developments, that seek to resolve this kind of problems, are in the process of finding the best solutions. The UC and power dispatch problems are among the most investigated related issues [13-14]. Accordingly, the UC problem should be modified in order to consider the uncertain characteristics of the WES in the power balance constraint.

\section{B. Related Work}

The UC problem has mostly been considered as a nonlinear mixed-integer optimization problem [5]. Priority List (PL), an earlier technique for solving the UC problem, has been based on sorting thermal units in a list according to their production costs in an increasing manner [13]. Units to be committed are extracted from the list in order to meet the hourly power demand. The PL method seems to be easy and can provide a quick pre-estimation of the optimum solution but it is criticized for its high convergence time and the large required number of units [14]. Several other strategies and formulations have been proposed to solve the UC problem such as dynamic programming (DP) [15], Lagrangian Relaxation (LR) [16-17], Benders Decomposition (BD) [18-19], Mixed-Integer Linear Programming (MILP) [20-22], neural networks [23-24], and meta-heuristic techniques [2, 25-28]. However, these strategies have certain limitations [4]. For example, DP-based approaches are considered as impractical for the case of large power networks. Compared with the DP method, the LR method is more effective because of its flexibility in managing various types of constraints. However, the coupling constraints are not often met by the dual optimum solution provided by the LR approach. Another limitation of the LR method is the sensitivity of the optimum solution of the UC problem to the Lagrange multipliers. Optimization methods based on the MILP approach have also been criticized for their high CPU

Corresponding author: Khalid Alqunun 
time especially when the power system is large in size [2]. Regarding the meta-heuristic based techniques, despite they can achieve hopeful results when their parameters are well tuned, they are not above criticism. One of their main disadvantages is the requirement of delicate tuning of the optimization algorithm parameters which is a fundamental step to ensure their effectiveness.

Nowadays, due to the improvements in the mathematical modeling solvers utilized in optimization problems, MILPbased models are becoming the most used for solving the UC problem. Two main features justified the use of the MILP in the modern optimization solvers: (i) the Mixed-Integer Programming (MIP) solver provides feasible solutions, and (ii) the level of optimality is controllable [29]. Instead of focusing on the optimization algorithm, nowadays solvers require excellent problem modeling. MIP solvers like CPLEX, GUROBI and XPRESS [30] have become quite popular in both academia and industry, especially after the year 2000. In recent years, several works regarded modeling and solving the Stochastic UC (SUC) including the WESs problem. The main contributions of these works are focused in the forecasting methods of WP. As WES is a fluctuating electrical energy source, the forecasts of wind power generation are useful for UC and dispatch problems [31]. In [32], a combined wavelet and time series methods based on short-term forecasting procedure for small farms was presented. An approach using chaos theories for prediction of wind speed and WP was proposed in [33]. Other prediction techniques based on artificial intelligence, such as neural networks [34-36], fuzzy logic systems [38] and evolutionary algorithms [38] have been also developed. According to the used methodology, forecasting procedures can be categorized into two categories: physical and statistical approaches. Physical approaches, also called deterministic approaches, are based on numerical weather data [39-40]. Statistical approaches use a historical data base to estimate the future wind speed and WP at any future time [41]. In recent years, more emphasis has been given to Probability Density Functions (PDFs) to describe the stochastic characteristics of wind speed [42-45]. One of the pioneer works in investigating power generation scheduling with WES is described in [43]. The authors used a stochastic programming approach called Here-and-Now (HN) strategy to model the stochastic optimization problem. Although, it is demonstrated that the impact of the random WP on the power dispatch can be easily evaluated when such a strategy is applied, the production cost has been approximated by a quadratic function.

According to [46], the HN strategy is used when the decision must be made even before the first realization of the random variables. The basic idea of this approach is to transform the stochastic problems into equivalent deterministic programs. In $\mathrm{HN}$ approach, the decision variables of the stochastic problem are detached from the random variables. Furthermore, all uncertain variables are described by chance constraints. PDFs and Cumulative Distribution Functions (CDFs) are used to convert these constraints into deterministic constraints. The Weibull distribution function has been widely used to describe the random wind speed and WP characteristics.

\section{Study Aims and Contribution}

Even though an enormous amount of research work has been conducted on the integration of WP resources into power systems, the UC problem with simultaneous integration of these devices is hardly studied. Thus, this study aims to develop an efficient model for the Chance-Constrained UC (CCUC) problem with both wind turbines and thermal generators, where the violation of the power balance constraint caused by the uncertain output power of the WESs is restricted by a user-defined probability. This probability represents the tolerance in which the power balance constraint cannot be satisfied at a given time $t$. In the problem formulation, all operating constraints and power system losses are considered. The HN strategy, used for modeling the economic dispatch [43] is extended for the CCUC problem formulation. Using that strategy, the CCUC problem is converted into a deterministic optimization problem. Weibull PDF is employed to describe the WP. The obtained deterministic problem is then implemented in GAMS interface and the CEPLEX MIP solver is employed for its solution. The effectiveness of the proposed strategy is evaluated on a six-unit system including wind farm. The results are compared with those obtained in other works.

\section{DETERMINISTIC UC PROBLEM FORMULATION}

Mostly, the UC problem is formulated as a minimization problem. It aims to meet the load profile over a planned timehorizon with minimum total operating cost subject to several equality and inequality constraints. The decision variables of the problem are the status of each unit which is a binary value and the power output of each online unit which is a continuous variable.

\section{A. Objective Function}

As given in (1), the principal components of the total operating cost of the thermal units are the fuel cost and the transitional costs which are start-up and shut-down costs [46]:

$$
\begin{aligned}
C_{T}= & {\left[\sum_{t=1}^{T} \sum_{i=1}^{N} C_{i}\left(P_{i}^{t}\right)\right] u_{i}^{t}+\left[\sum_{t=1}^{T} \sum_{i=1}^{N} S_{i}^{t}\right] u_{i}^{t}\left(1-u_{i}^{t}\right) } \\
& +\left[\sum_{t=1}^{T} \sum_{i=1}^{N} D_{i}^{t}\right] u_{i}^{t}\left(1-u_{i}^{t}\right)
\end{aligned}
$$

The fuel cost of the $i$-th unit at time $t$ depends on its real output power at the same time and it can be approximated by:

$$
C_{i}\left(P_{i}^{t}\right)=a_{i}+b_{i} P_{i}^{t}+c_{i}\left(P_{i}^{t}\right)^{2}
$$

where, $a_{i}, b_{i}$, and $c_{i}$ are the cost coefficients of the $i$-th unit. $N$ is the number of thermal units, $T$ is the scheduling period in hours, $P_{i}^{t}$ is the generation in MW of unit $i$ at time $t, u_{i}^{t}$ is the status of unit $i$ at time $t$ ( 1 for ON and 0 for OFF), and $S_{i}^{t}$ and $D_{i}^{t}$ are start-up and shut-down costs of unit $i$ at time $t$.

The start-up cost is the required cost to restart the unit from the OFF status to the ON status. It comprises mainly of the reheating and maintenance costs. That cost depends on the offline duration preceding the start-up. Several expressions such as two-valued staircase, linear and exponential-based 
functions have been proposed [4] to describe the start-up cost. The first function adopted in this paper is:

$$
S_{i}^{t}=\left\{\begin{array}{l}
S_{h i} \text { if } T_{i, \text { OFF }}^{t} \leq T_{i}^{D}+T_{i}^{C} \\
S_{c i} \text { if } T_{i, \text { OFF }}^{t}>T_{i}^{D}+T_{i}^{C}
\end{array}\right.
$$

where $S_{h i}$ and $S_{c i}$ are the hot start-up and cold start-up costs of unit $i$ respectively, $T_{i, O F F}^{t}$ is the duration in hours for which the unit $i$ is continuously OFF at time $t$, and $T_{i}^{D}$ and $T_{i}^{C}$ are minimum down time and minimum up time in hours of unit $i$ respectively.

The transition cost of thermal units from the online status to the offline status may be considered constant and it does not depend on the online duration preceding the shut-down. However, it is often ignored in the UC problem formulations.

\section{B. Deterministic Constraints}

Four operating constraints are taken more frequently into account in the classical UC problem.

\section{1) Power Balance Constraint}

At each hour $t$, the total generated power must meet the predicted load $P_{D}^{t}$ plus the total real losses $P_{L}^{t}$ as described by (4):

$$
\sum_{i=1}^{N}\left(P_{i}^{t}\right) u_{i}^{t}-P_{D}^{t}-P_{L}^{t}=0
$$

\section{2) Unit Limits}

The output power $P_{i}^{t}$ of the $i$-th committed unit must be within its lower and upper limits.

$$
P_{i}^{\min } \leq P_{i}^{t} \leq P_{i}^{\max }
$$

\section{3) Minimum Up/Down Times}

These constraints signify the minimum required durations for which the de-committed/committed unit can be turned ON/OFF. $T_{i, O N}^{t}$ is the duration in hours for which the unit $i$ is continuously $\mathrm{ON}$ at time $t$.

$$
\left\{\begin{array}{l}
T_{i, \text { ON }}^{t}>T_{i}^{U} \\
T_{i, \text { OFF }}^{t}>T_{i}^{D}
\end{array}\right.
$$

\section{4) Spinning Reserve Constraints}

Spinning reserve is the reserve capacity of all units synchronized on the grid and ready to take load. At each hour $t$, the spinning reserve constraint is described as:

$$
\sum_{i=1}^{N} P_{i}^{t} u_{i}^{t} \geq P_{D}^{t}+S R^{t}
$$

where $S R^{t}$ is the system spinning reserve at time $t$.

\section{EQUIVALENT CHANCE-CONSTRAINED UC MODEL}

The output power of any WES depends on wind speed and the technical characteristics of the turbine. Moreover, wind speed is influenced by several factors depending on the geographical location. Indeed, wind speed uncertainty may be characterized by probability principles [43]. The incorporation of WES in the UC problem can be described by including new constraints into the classic UC model. These constraints describe the stochastic characteristic of wind speed that obviously influences the output power of the WES. In this study, the following stochastic constraint is included in the CCUC problem formulation:

$$
\operatorname{Pr}\left(\sum_{i=1}^{N} P_{i}^{t}+W^{t} \leq P_{D}^{t}+P_{L}^{t}\right) \leq \sigma
$$

where $\sigma$ is a user-defined parameter in the range $[0,1]$ that represents the tolerance that power balance constraint cannot be satisfied at a given time $t, P_{D}^{t}$ is the total power demand and $P_{L}^{t}$ is the total system losses calculated using $B$-loss formula [13]. $W^{t}$ is the output power of the WES at hour $t$. Obviously, power balance constraint (4) is replaced by the following equation, which results from the integration of the WES:

$$
\sum_{i=1}^{N}\left(P_{i}^{t}\right) u_{i}^{t}+W^{t}-P_{D}^{t}-P_{L}^{t}=0
$$

The probability distribution has been widely used to describe the stochastic characteristics of wind speed [44]. In this study, the two-parameter Weibull PDF described by (10) is proposed to approximate wind speed distribution. The probability to have wind speed equal to or less than $v$, called $\mathrm{CDF}$ is given in (11).

$$
\begin{array}{r}
f_{V}(v)=\frac{k}{c}\left(\frac{v}{c}\right)^{k-1} \exp \left[-\left(\frac{v}{c}\right)^{k}\right] \\
F_{V}(v)=\int_{0}^{v} f_{V}(\tau) d \tau=1-\exp \left(-\left(\frac{v}{c}\right)^{k}\right), v \geq 0
\end{array}
$$

where $v$ is the wind speed. Parameters $k$ and $c$ are the shape and scale factors which depend on the geographical location of the wind turbine.

After predicting wind velocity from the Weibull PDF, the output power $W$ of the wind turbine can be determined as:

$$
\begin{gathered}
W=\phi(V)=0, \text { if } V<v_{\text {in }} \text { or } V>v_{\text {out }} \\
W=\phi(V)=\frac{\left(V-v_{\text {in }}\right) w_{r}}{v_{r}-v_{\text {in }}} \text { if } v_{\text {in }} \leq V<v_{r} \\
W=\phi(V)=w_{r}, \text { if } v_{r} \leq V<v_{\text {out }}
\end{gathered}
$$

where $v_{\text {in }}, v_{\text {out }}$ and $v_{r}$ are cut-in, cut-out, and rated wind speeds respectively.

Using probability theories and (12)-(14), the probability to have a WES between zero and rated power can be described as [43]: 


$$
\begin{aligned}
F_{W}(w) & =\operatorname{Pr}(W \leq w) \\
& =1-\exp \left\{-\left(\frac{\left(1+\frac{h w}{w_{r}}\right) v_{\text {in }}}{c}\right)^{k}\right\}+\exp \left(-\left(\frac{v_{\text {out }}}{c}\right)^{k}\right)
\end{aligned}
$$

where $h=\frac{v_{r}-v_{i n}}{v_{i n}}$.

From (15), the stochastic constraint given in (8) can be converted into the following deterministic constraint:

$$
\operatorname{Pr}\left\{W^{t} \leq P_{D}^{t}+P_{L}^{t}-\sum_{i=1}^{N} P_{i}^{t}\right\}=F_{W}\left(P_{D}^{t}+P_{L}^{t}-\sum_{i=1}^{N} P_{i}\right) \leq \sigma
$$

\section{IMPLEMENTATION OF THE SOLVING METHOD}

In order to have minimum fuel cost, the output power of the wind turbine must be maximum as much as possible. However, it is required to make sure that this power meets the constraint in (16). To meet this objective, for each time-horizon $t$, wind power is increased gradually until constraint (16) is not verified or until the maximum generation of the wind turbine is reached. Then, the total generation will be subdivided economically between all thermal units in order to cover the total load power $P_{D}^{t}$ plus the total losses for the same timehorizon minus the suggested wind turbine output $W^{t}$. The MILP is used in this study to model the CCUC problem. In order to apply the MILP solvers effectively, nonlinearities must be removed from the problem. Therefore, the quadratic form of the fuel cost of thermal units is converted into piecewise linear functions. The CCUC problem is implemented in GAMS and the CEPLEX MIP solver is employed for its solution.

\section{CASE STUDY}

To test the performance of the proposed strategy in solving the stochastic UC problem incorporating both thermal generation units and WES, two test cases are considered in this study (all system data are taken from http://motor.ece.iit.edu/ data): (a)Case 1: 6-bus system without WES and (b) Case 2: 6bus system with WES

\section{A. Case 1: 6-Bus System without WES}

As given in Figure 1, the 6-bus system consists of three thermal generating units, seven transmission lines and three loads.

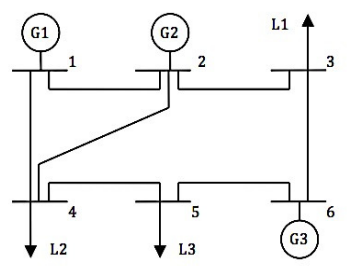

Fig. 1. The 6-bus system.

The generating units and the transmission line characteristics are shown in Tables I and II. The hourly demand is illustrated in Table III. The objective is to deliver the required energy with the minimum operation cost, and assuming wind power is unavailable in this case. The network operator would satisfy the demand whilst taking into consideration the power supply limitations and the transmission line's constraints. The optimum hourly power supply of the three generating units obtained after the convergence of the optimization algorithm is depicted in Figure 2. It is clear that Unit 1 supplies the majority of the required demand due to its high capacity and low production cost.

TABLE I. UNIT DATA

\begin{tabular}{|c|c|c|c|c|}
\hline & Unit 1 & Unit 2 & Unit 3 \\
\hline \multicolumn{2}{|c|}{ Pmin (MW) } & 100 & 10 & 10 \\
\hline \multicolumn{2}{|c|}{ Pmax (MW) } & 220 & 200 & 50 \\
\hline \multirow{3}{*}{ Cost coefficients } & $\mathbf{c}(\$ / M W 2 h)$ & 0.014 & 0.02 & 0.086 \\
\cline { 2 - 5 } & $\mathbf{b}$ (\$/MWh) & 19.96 & 23 & 29.14 \\
\cline { 2 - 5 } & $\mathbf{a ~ ( \$ ) ~}$ & 200 & 150 & 50 \\
\hline \multicolumn{2}{|c|}{ Start-up cost (\$) } & 50 & 40 & 0 \\
\hline \multicolumn{2}{|c|}{ Shut-down cost (\$) } & 100 & 200 & 0 \\
\hline \multicolumn{2}{|c|}{ Minimum up time (h) } & 4 & 3 & 1 \\
\hline \multicolumn{2}{|c|}{ Minimum down time (h) } & 4 & 2 & 1 \\
\hline \multicolumn{2}{|c|}{ Ramp up rate (MW/h) } & 40 & 30 & 20 \\
\hline \multicolumn{2}{|c|}{ Ramp down rate (MW/h) } & 50 & 35 & 20 \\
\hline
\end{tabular}

TABLE II. LINE DATA

\begin{tabular}{|c|c|c|c|c|}
\hline Line & From & To & $\boldsymbol{X}(\mathbf{p u})$ & Flow limit (MW) \\
\hline 1 & 1 & 2 & 0.17 & 140 \\
\hline 2 & 1 & 4 & 0.258 & 110 \\
\hline 3 & 2 & 4 & 0.197 & 140 \\
\hline 4 & 5 & 6 & 0.14 & 140 \\
\hline 5 & 3 & 6 & 0.018 & 130 \\
\hline 6 & 2 & 3 & 0.037 & 150 \\
\hline 7 & 4 & 5 & 0.037 & 50 \\
\hline
\end{tabular}

TABLE III. HOURLY DEMAND IN MW

\begin{tabular}{|c|c|c|c|}
\hline Hour & Demand & Hour & Demand \\
\hline 1 & 170 & 13 & 236.1 \\
\hline 2 & 175.19 & 14 & 242.18 \\
\hline 3 & 165.15 & 15 & 243.6 \\
\hline 4 & 158.67 & 16 & 248.86 \\
\hline 5 & 154.73 & 17 & 255.79 \\
\hline 6 & 155.06 & 18 & 256 \\
\hline 7 & 160.48 & 19 & 246.74 \\
\hline 8 & 173.39 & 20 & 245.97 \\
\hline 9 & 177.6 & 21 & 237.35 \\
\hline 10 & 186.81 & 22 & 237.31 \\
\hline 11 & 206.96 & 23 & 232.67 \\
\hline 12 & 228.61 & 24 & 195.93 \\
\hline
\end{tabular}

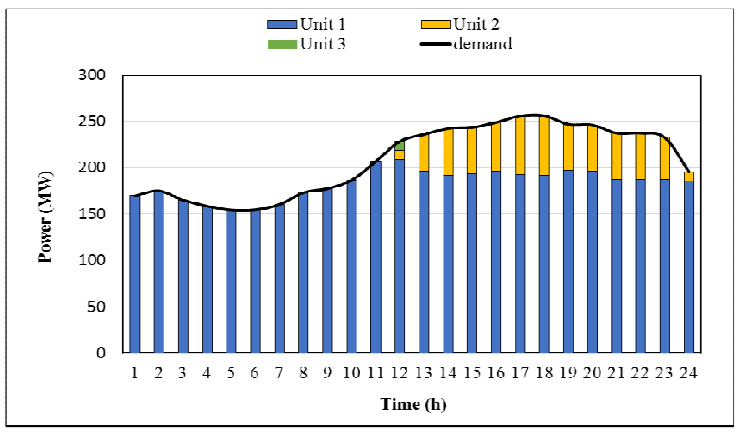

Fig. 1. Power supply in Case 1 (without wind power). 
TABLE IV. OPERATION COST (\$) OF GENERATING UNITS IN CASE 1

\begin{tabular}{|c|c|c|c|c|}
\hline Time (h) & Unit 1 & Unit 2 & Unit 3 & Total \\
\hline 1 & 4010 & & & 4010 \\
\hline 2 & 4139.75 & & & 4139.75 \\
\hline 3 & 3888.75 & & & 3888.75 \\
\hline 4 & 3733.08 & & & 3733.08 \\
\hline 5 & 3638.52 & & & 3638.52 \\
\hline 6 & 3646.44 & & & 3646.44 \\
\hline 7 & 3776.52 & & & 3776.52 \\
\hline 8 & 4094.75 & & & 4094.75 \\
\hline 9 & 4200 & & & 4200 \\
\hline 10 & 4430.25 & & & 4430.25 \\
\hline 11 & 4934 & & & 4934 \\
\hline 12 & 4975.25 & 590 & 350 & 5915.25 \\
\hline 13 & 4662.5 & 1110 & & 5772.5 \\
\hline 14 & 4564.5 & 1350 & & 5914.5 \\
\hline 15 & 4600 & 1350 & & 5950 \\
\hline 16 & 4668.965 & 1415.036 & & 6084.001 \\
\hline 17 & 4567.319 & 1700.928 & & 6268.247 \\
\hline 18 & 4564.239 & 1709.592 & & 6273.831 \\
\hline 19 & 4678.5 & 1350 & & 6028.5 \\
\hline 20 & 4659.25 & 1350 & & 6009.25 \\
\hline 21 & 4443.75 & 1350 & & 5793.75 \\
\hline 22 & 4442.75 & 1350 & & 5792.75 \\
\hline 23 & 4451.75 & 1230 & & 5681.75 \\
\hline 24 & 4408.25 & 390 & & 4798.25 \\
\hline Total & 104179.083 & 16245.556 & 350 & 120774.639 \\
\hline
\end{tabular}

Unit 1 is committed alone at hours 1-11 since the hourly demand is less than its maximum capacity of $22 \mathrm{MW}$. At hours $1-11$, the hourly demand variation does not exceed the ramping limit of Unit 1. Unit 3 does not have start-up/shut-down costs, while these costs are very high in Unit 2. Therefore, Unit 3 has been specifically turned on at hour 12 with Unit 2 to reduce the operation cost. The hourly operation cost is shown in Table IV. The operation cost begins with $\$ 4010$ when Unit 1 was only operated at hour 1. However, the operation cost is increased to $\$ 6273$ at the peak time (hour 18), which represents an increase of $56 \%$ in the total cost as compared with hour 1 .

\section{B. Case 2: 6-Bus System with WES}

In this case, the 6-bus system of Case 1 with an extra wind farm is used. The wind farm comprises of 50 WESs of the same parameters as shown in Table V. The hourly availability of wind power in MW of the wind farm is depicted in Table VI. For the applicability of the problem, tolerance $\sigma$ should verify the following inequality [43] which ensures $\sigma \in[0.1447,1]$ :

$$
\operatorname{Pr}(W=0) \leq \sigma<1
$$

TABLE V. WIND PARAMETERS

\begin{tabular}{|c|c|c|c|c|}
\hline $\boldsymbol{K}$ & $\boldsymbol{C}$ & $\boldsymbol{v}_{\text {in }}$ & $\boldsymbol{v}_{\text {out }}$ & $\boldsymbol{v}_{\boldsymbol{r}}$ \\
\hline 1.7 & 15 & 5 & 45 & 15 \\
\hline
\end{tabular}

TABLE VI. HOURLY WIND POWER IN MW

\begin{tabular}{|c|c|c|c|}
\hline Hour & Wind power & Hour & Wind power \\
\hline 1 & 44 & 13 & 84 \\
\hline 2 & 70.2 & 14 & 80 \\
\hline 3 & 76 & 15 & 78 \\
\hline 4 & 82 & 16 & 32 \\
\hline 5 & 84 & 17 & 4 \\
\hline 6 & 84 & 18 & 0 \\
\hline 7 & 100 & 19 & 10 \\
\hline 8 & 100 & 20 & 0 \\
\hline 9 & 78 & 21 & 6 \\
\hline 10 & 64 & 22 & 56 \\
\hline 11 & 100 & 23 & 82 \\
\hline 12 & 92 & 24 & 52 \\
\hline
\end{tabular}

TABLE VII. POWER SUPPLY OF THE GENERATING UNITS (MW) IN CASE 2 WITH VARIABLE WIND TOLERANCE

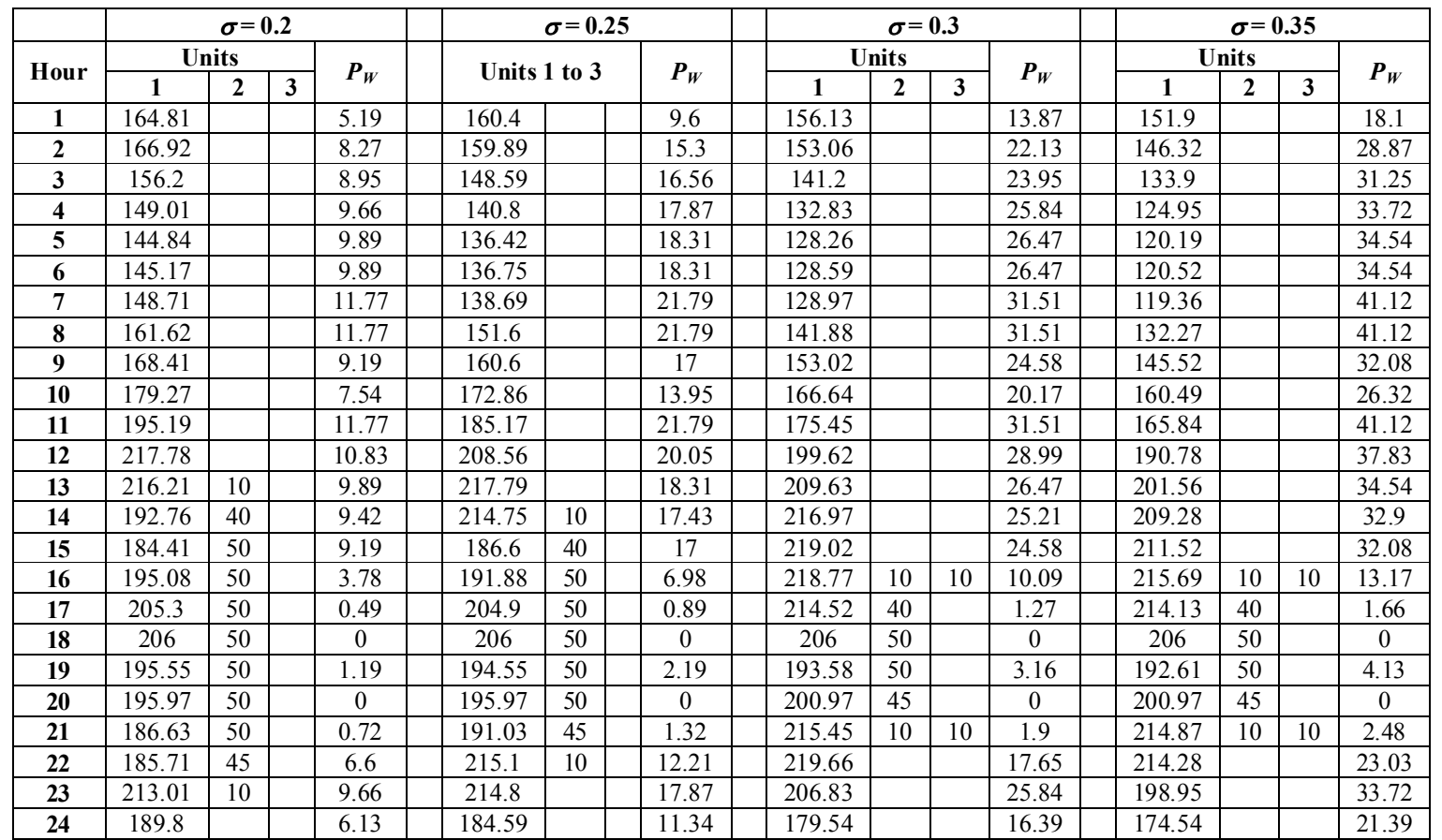


The problem is solved for various values of the threshold tolerance $\sigma$. In this case, $\sigma$ is changing from 0.2 to 0.35 . The injection of wind power has completely changed the power production of the generating units. The impact of wind power $\left(P_{W}\right)$ on the supply of the generating units is demonstrated in Table VII. It is clear that the penetration rate of wind energy increases if the tolerance increases and vice-versa. The results obtained for the proposed approach are compared with those found for the stochastic security-constrained unit commitment (SSCUC) investigated in [46]. From Table VIII, it is clear that the proposed strategy provides minimum cost when a wind farm is integrated. Moreover, if the tolerance increases the production cost decreases thanks to the increase of the WP output.

TABLE VIII. TOTAL PRODUCTION COSTS

\begin{tabular}{|c|c|c|c|c|c|}
\hline \multirow{2}{*}{ Method } & \multicolumn{4}{|c|}{ Proposed approach } & \multirow{2}{*}{ SSCUC [46] } \\
\cline { 2 - 6 } & $\boldsymbol{\sigma}=\mathbf{0 . 2}$ & $\boldsymbol{\sigma}=\mathbf{0 . 2 5}$ & $\boldsymbol{\sigma}=\mathbf{0 . 3}$ & $\boldsymbol{\sigma}=\mathbf{0 . 3 5}$ & \\
\hline Total cost (\$) & 116,203 & 112,418 & 108,845 & 105,415 & $121,445.015$ \\
\hline
\end{tabular}

\section{CONCLUSION}

Due to the high emergence of renewable energy sources in nowadays power grids, it is important to take into account the intermittent characteristics of these sources. A strategy for solving the UC problem comprising uncertain production is proposed in this paper. In order to address the uncertainty of the output of the WES, the power balance constraint is represented as a chance-constraint with a pre-defined probability called tolerance. The stochastic UC problem is reformulated as a deterministic mixed-integer optimization problem. To do so, the HN strategy was used to convert the chance constraint into a deterministic constraint. The applicability of the proposed method is tested on the six-unit system including wind farms. The simulation results show that with low values of tolerance both systems (with and without WES) are equivalent. Moreover, the more the tolerance increases, the more wind power penetration increases and, accordingly, fuel cost decreases.

\section{REFERENCES}

[1] B. Saravanan, E. R. Vasudevan, and D. P. Kothari, "Unit commitment problem solution using invasive weed optimization algorithm," International Journal of Electrical Power \& Energy Systems, vol. 55, pp. 21-28, Feb. 2014, doi: 10.1016/j.ijepes.2013.08.020.

[2] D. F. Rahman, A. Viana, and J. P. Pedroso, "Metaheuristic search based methods for unit commitment," International Journal of Electrical Power \& Energy Systems, vol. 59, pp. 14-22, Jul. 2014, doi: 10.1016/ j.ijepes.2014.01.038.

[3] M. Haberg, "Fundamentals and recent developments in stochastic unit commitment," International Journal of Electrical Power \& Energy Systems, vol. 109, pp. 38-48, Jul. 2019, doi: 10.1016/j.ijepes. 2019.01.037.

[4] K.-H. Jo and M.-K. Kim, "Stochastic Unit Commitment Based on MultiScenario Tree Method Considering Uncertainty," Energies, vol. 11, no. 4, Apr. 2018, doi: 10.3390/en11040740, Art. no. 740.

[5] V. K. Tumuluru, Z. Huang, and D. H. K. Tsang, "Unit commitment problem: A new formulation and solution method," International Journal of Electrical Power \& Energy Systems, vol. 57, pp. 222-231, May 2014, doi: 10.1016/j.ijepes.2013.11.043.

[6] A. Torchani, A. Boudjemline, H. Gasmi, Y. Bouazzi, and T. Guesmi, "Dynamic Economic/Environmental Dispatch Problem Considering
Prohibited Operating Zones," Engineering, Technology \& Applied Science Research, vol. 9, no. 5, pp. 4586-4590, Oct. 2019.

[7] G. Morales-Espana, C. Gentile, and A. Ramos, "Tight MIP formulations of the power-based unit commitment problem," OR Spectrum, vol. 37, no. 4, pp. 929-950, Oct. 2015, doi: 10.1007/s00291-015-0400-4.

[8] F. H. Aghdam and M. T. Hagh, "Security Constrained Unit Commitment (SCUC) formulation and its solving with Modified Imperialist Competitive Algorithm (MICA)," Journal of King Saud University Engineering Sciences, vol. 31, no. 3, pp. 253-261, Jul. 2019, doi: 10.1016/j.jksues.2017.08.003.

[9] S. Atakan, G. Lulli, and S. Sen, "A State Transition MIP Formulation for the Unit Commitment Problem," IEEE Transactions on Power Systems, vol. 33, no. 1, pp. 736-748, Jan. 2018, doi: 10.1109/TPWRS. 2017.2695964 .

[10] I. Marouani, A. Boudjemline, T. Guesmi, and H. H. Abdallah, “A Modified Artificial Bee Colony for the Non-Smooth Dynamic Economic/Environmental Dispatch," Engineering, Technology \& Applied Science Research, vol. 8, no. 5, pp. 3321-3328, Oct. 2018.

[11] P. de Jong, A. Kiperstok, and E. A. Torres, "Economic and environmental analysis of electricity generation technologies in Brazil," Renewable and Sustainable Energy Reviews, vol. 52, pp. 725-739, Dec. 2015, doi: 10.1016/j.rser.2015.06.064.

[12] G. Morales-Espana, A. Lorca, and M. M. de Weerdt, "Robust unit commitment with dispatchable wind power," Electric Power Systems Research, vol. 155, pp. 58-66, Feb. 2018, doi: 10.1016/j.epsr. 2017.10.002.

[13] R. C. Johnson, H. H. Happ, and W. J. Wright, "Large Scale HydroThermal Unit Commitment-Method and Results," IEEE Transactions on Power Apparatus and Systems, vol. PAS-90, no. 3, pp. 1373-1384, May 1971, doi: 10.1109/TPAS.1971.292941.

[14] I. G. Damousis, A. G. Bakirtzis, and P. S. Dokopoulos, "A solution to the unit-commitment problem using integer-coded genetic algorithm," IEEE Transactions on Power Systems, vol. 19, no. 2, pp. 1165-1172, May 2004, doi: 10.1109/TPWRS.2003.821625.

[15] W. L. Snyder, H. D. Powell, and J. C. Rayburn, "Dynamic Programming Approach to Unit Commitment," IEEE Transactions on Power Systems, vol. 2, no. 2, pp. 339-348, May 1987, doi: 10.1109/ TPWRS.1987.4335130.

[16] S. Virmani, E. C. Adrian, K. Imhof, and S. Mukherjee, "Implementation of a Lagrangian relaxation based unit commitment problem," IEEE Transactions on Power Systems, vol. 4, no. 4, pp. 1373-1380, Nov. 1989, doi: 10.1109/59.41687.

[17] D. Murata and S. Yamashiro, "Unit commitment scheduling by Lagrange relaxation method taking into account transmission losses," Electrical Engineering in Japan, vol. 152, no. 4, pp. 27-33, 2005, doi: 10.1002/eej.20119.

[18] H. Ma and S. M. Shahidehpour, "Transmission-constrained unit commitment based on Benders decomposition," International Journal of Electrical Power \& Energy Systems, vol. 20, no. 4, pp. 287-294, May 1998, doi: 10.1016/S0142-0615(97)00058-6.

[19] J. Alemany and F. Magnago, "Benders decomposition applied to security constrained unit commitment: Initialization of the algorithm," International Journal of Electrical Power \& Energy Systems, vol. 66, pp. 53-66, Mar. 2015, doi: 10.1016/j.ijepes.2014.10.044.

[20] M. Carrion and J. M. Arroyo, "A computationally efficient mixedinteger linear formulation for the thermal unit commitment problem," IEEE Transactions on Power Systems, vol. 21, no. 3, pp. 1371-1378, Aug. 2006, doi: 10.1109/TPWRS.2006.876672.

[21] J. Ostrowski, M. F. Anjos, and A. Vannelli, "Tight Mixed Integer Linear Programming Formulations for the Unit Commitment Problem," IEEE Transactions on Power Systems, vol. 27, no. 1, pp. 39-46, Feb. 2012, doi: 10.1109/TPWRS.2011.2162008.

[22] J. Alemany, L. Kasprzyk, and F. Magnago, "Effects of binary variables in mixed integer linear programming based unit commitment in largescale electricity markets," Electric Power Systems Research, vol. 160, pp. 429-438, Jul. 2018, doi: 10.1016/j.epsr.2018.03.019.

[23] A. A. Girgis and S. Varadan, "Unit commitment using load forecasting based on artificial neural networks," Electric Power Systems Research, 
vol. 32, no. 3, pp. 213-217, Mar. 1995, doi: 10.1016/0378-7796(94) 00917-S.

[24] M. Z. Jahromi, M. M. Hosseini Bioki, M. Rashidinejad, and R. Fadaeinedjad, "Solution to the unit commitment problem using an artificial neural network," Turkish Journal of Electrical Engineering \& Computer Sciences, vol. 21, pp. 198-212, 2013.

[25] F. Barani, M. Mirhosseini, H. Nezamabadi-pour, and M. M. Farsangi, "Unit commitment by an improved binary quantum GSA," Applied Soft Computing, vol. 60, pp. 180-189, Nov. 2017, doi: 10.1016/ j.asoc.2017.06.051.

[26] M. Nemati, M. Braun, and S. Tenbohlen, "Optimization of unit commitment and economic dispatch in microgrids based on genetic algorithm and mixed integer linear programming," Applied Energy, vol. 210, pp. 944-963, Jan. 2018, doi: 10.1016/j.apenergy.2017.07.007.

[27] L. A. C. Roque, D. B. M. M. Fontes, and F. A. C. C. Fontes, "A multiobjective unit commitment problem combining economic and environmental criteria in a metaheuristic approach," Energy Procedia, vol. 136, pp. 362-368, Oct. 2017, doi: 10.1016/j.egypro.2017.10.290.

[28] S. R. K, L. Panwar, B. K. Panigrahi, and R. Kumar, "Binary whale optimization algorithm: a new metaheuristic approach for profit-based unit commitment problems in competitive electricity markets," Engineering Optimization, vol. 51, no. 3, pp. 369-389, Mar. 2019, doi: 10.1080/0305215X.2018.1463527.

[29] S. M. Pour, J. H. Drake, L. S. Ejlertsen, K. M. Rasmussen, and E. K. Burke, "A hybrid Constraint Programming/Mixed Integer Programming framework for the preventive signaling maintenance crew scheduling problem," European Journal of Operational Research, vol. 269, no. 1, pp. 341-352, Aug. 2018, doi: 10.1016/j.ejor.2017.08.033.

[30] R. Anand, D. Aggarwal, and V. Kumar, "A comparative analysis of optimization solvers," Journal of Statistics and Management Systems, vol. 20, no. 4, pp. 623-635, Jul. 2017, doi: 10.1080/09720510. 2017.1395182.

[31] A. M. Foley, P. G. Leahy, A. Marvuglia, and E. J. McKeogh, "Current methods and advances in forecasting of wind power generation," Renewable Energy, vol. 37, no. 1, pp. 1-8, Jan. 2012, doi: 10.1016/ j.renene.2011.05.033.

[32] H. Liu, H.-Q. Tian, C. Chen, and Y. Li, "A hybrid statistical method to predict wind speed and wind power," Renewable Energy, vol. 35, no. 8, pp. 1857-1861, Aug. 2010, doi: 10.1016/j.renene.2009.12.011.

[33] M. Hayashi and K. Nagasaka, "Wind speed prediction and determination of wind power output with multi-area weather data by deterministic chaos," in International Conference on Advanced Mechatronic Systems, Kumamoto, Japan, Aug. 2014, pp. 192-197, doi: 10.1109/ICAMechS. 2014.6911649.

[34] U. B. Filik and T. Filik, "Wind Speed Prediction Using Artificial Neural Networks Based on Multiple Local Measurements in Eskisehir," Energy Procedia, vol. 107, pp. 264-269, Feb. 2017, doi: 10.1016/j.egypro. 2016.12.147.

[35] S. K. Paramasivan and D. Lopez, "Forecasting of Wind Speed using Feature Selection and Neural Networks," International Journal of Renewable Energy Research, vol. 6, no. 3, pp. 833-837, Sep. 2016.

[36] F. Fazelpour, N. Tarashkar, and M. A. Rosen, "Short-term wind speed forecasting using artificial neural networks for Tehran, Iran," International Journal of Energy and Environmental Engineering, vol. 7, no. 4, pp. 377-390, Dec. 2016, doi: 10.1007/s40095-016-0220-6.

[37] B. Zhu, M. Chen, N. Wade, and L. Ran, "A prediction model for wind farm power generation based on fuzzy modeling," Procedia Environmental Sciences, vol. 12, pp. 122-129, Jan. 2012, doi: 10.1016/j.proenv.2012.01.256

[38] R. Jursa and K. Rohrig, "Short-term wind power forecasting using evolutionary algorithms for the automated specification of artificial intelligence models," International Journal of Forecasting, vol. 24, no. 4, pp. 694-709, Oct. 2008, doi: 10.1016/j.ijforecast.2008.08.007.

[39] J. Sillmann et al., "Understanding, modeling and predicting weather and climate extremes: Challenges and opportunities," Weather and Climate Extremes, vol. 18, pp. 65-74, Dec. 2017, doi: 10.1016/j.wace. 2017.10.003.
[40] L. Landberg, "A mathematical look at a physical power prediction model," Wind Energy, vol. 1, no. 1, pp. 23-28, 1998, doi: 10.1002/(SICI)1099-1824(199809)1:1<23::AID-WE9>3.0.CO;2-9.

[41] G. Sideratos and N. D. Hatziargyriou, "An Advanced Statistical Method for Wind Power Forecasting," IEEE Transactions on Power Systems, vol. 22, no. 1, pp. 258-265, Feb. 2007, doi: 10.1109/TPWRS. 2006.889078 .

[42] R. Dai, Q. Qi, and J. D. McCalley, "Stochastic unit commitment for wind power interconnected system reserve requirement estimation," in IEEE Power Energy Society General Meeting, Chicago, IL, USA, Jul. 2017, pp. 1-5, doi: 10.1109/PESGM.2017.8274483.

[43] X. Liu and W. Xu, "Economic Load Dispatch Constrained by Wind Power Availability: A Here-and-Now Approach," IEEE Transactions on Sustainable Energy, vol. 1, no. 1, pp. 2-9, Apr. 2010, doi: 10.1109/ TSTE.2010.2044817.

[44] J. Hetzer, D. C. Yu, and K. Bhattarai, "An Economic Dispatch Model Incorporating Wind Power," IEEE Transactions on Energy Conversion, vol. 23, no. 2, pp. 603-611, Jun. 2008, doi: 10.1109/TEC.2007.914171.

[45] M. R. Patel, Wind and Solar Power Systems: design, analysis, and operation, Second Edition. London, UK: Taylor \& Francis Group, 2006.

[46] G. Haddadian, N. Khalili, M. Khodayar, and M. Shahidehpour, "Optimal coordination of variable renewable resources and electric vehicles as distributed storage for energy sustainability," Sustainable Energy, Grids and Networks, vol. 6, pp. 14-24, Jun. 2016, doi: 10.1016/ j.segan.2015.12.001. 